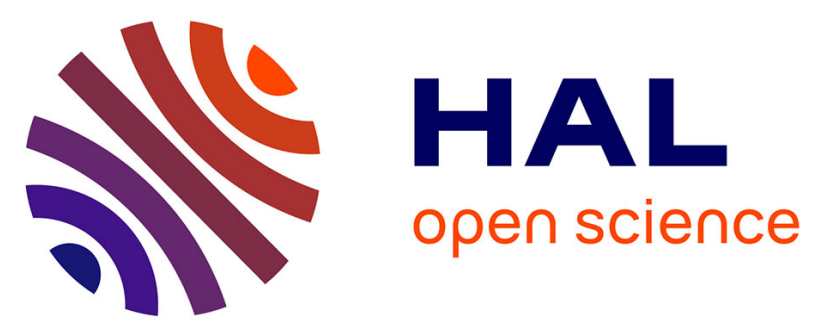

\title{
Modeling Hot Tearing during Solidification of Steels: Assessment and Improvement of Macroscopic Criteria through the Analysis of Two Experimental Tests
}

\author{
Michel Bellet, Olivier Cerri, M. Bobadilla, Yvan Chastel
}

\section{- To cite this version:}

Michel Bellet, Olivier Cerri, M. Bobadilla, Yvan Chastel. Modeling Hot Tearing during Solidification of Steels: Assessment and Improvement of Macroscopic Criteria through the Analysis of Two Experimental Tests. Metallurgical and Materials Transactions A, 2009, 40 (11), pp.Pages 2705-2717. 10.1007/s11661-009-9955-5 . hal-00487265

\section{HAL Id: hal-00487265}

https://hal-mines-paristech.archives-ouvertes.fr/hal-00487265

Submitted on 9 Mar 2011

HAL is a multi-disciplinary open access archive for the deposit and dissemination of scientific research documents, whether they are published or not. The documents may come from teaching and research institutions in France or abroad, or from public or private research centers.
L'archive ouverte pluridisciplinaire HAL, est destinée au dépôt et à la diffusion de documents scientifiques de niveau recherche, publiés ou non, émanant des établissements d'enseignement et de recherche français ou étrangers, des laboratoires publics ou privés. 


\title{
Modeling Hot Tearing during Solidification of Steels: Assessment and Improvement of Macroscopic Criteria through the Analysis of Two Experimental Tests
}

\author{
M. BELLET, O. CERRI, M. BOBADILLA and Y. CHASTEL \\ Hot tearing is an unacceptable defect found in products and parts obtained by solidification processes such as ingot \\ and continuous casting. It consists of the development of cracks during solidification, in regions which are not \\ completely solidified, more precisely in areas of mushy zones with high fraction of solid (typically 0.9 and beyond), \\ when the material undergoes deformations associated with tensile stress. In this study, two hot tearing tests have \\ been studied in order to evaluate the predictive capability of several macroscopic criteria published in the literature. \\ The first test is a new test specifically designed for constrained shrinkage by the present authors, while the second \\ test is an ingot bending test developed in the eighties. For both tests, a thermal-mechanical analysis is performed, in \\ order to provide the key variables for the different selected criteria. A comparison with experimental results allows \\ to make a critical assessment of those criteria regarding their ability to predict crack occurrence. The criterion \\ initially proposed by Won et al. [8] has been found to be the best suited for the prediction of solidification cracking. \\ As this criterion is essentially based on the "brittle temperature range", critical considerations regarding non- \\ equilibrium solidification have led to suggest an extension of this criterion. This new macroscopic criterion \\ improves the prediction capacity.
}

\section{INTRODUCTION}

Hot tearing arises from a complex combination of thermalmechanical and solidification phenomena. This solidification defect is basically associated with incomplete liquid feeding and tensile stress generated in coherent regions of mushy zones, more specifically areas with high solid fraction of 0.9 and beyond [1]. Many authors have reported both strength and ductility drop in a critical interval of high solid fraction (brittle temperature range, BTR). Indeed, interdendritic residual liquid films act as weak zones when the semi-solid material is submitted to a mechanical loading. Consequently, the spatial distribution of the liquid phase in between the solid dendritic arms has a first order influence with respect to crack initiation together with the wetting of the solid phase by the liquid one, which is controlled by surface tension.

A schematic summary of the concurrent phenomena leading to hot tearing is given in Figure 1. Regions with solid fraction over the coherency point (for which the solid skeleton is continuous, and then capable to stand stresses) become less permeable to the liquid phase. Then the possible cavities created either by solidification shrinkage or by rupture of residual interdendritic liquid films cannot be compensated by liquid feeding, which leads to crack initiation. This explains that for solid fractions higher than $g_{s}^{\text {cohe }}$ the ductility limit $\mathcal{E}_{R}$ decreases. At the coalescence point, more and more solid bridges form and interdendritic liquid pockets are isolated. For higher solid fractions, some cracks may still be initiated but this phenomenon is limited because of solid bridging and grain coalescence. This explains that for solid fractions greater than

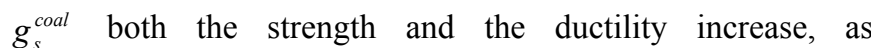
solidification proceeds. All together, those phenomena result in a ductility trough which is often interpreted as a brittleness interval and can be defined either in terms of critical solid fractions or critical temperatures (the above mentioned BTR).

Depending on solidification conditions and materials, hot tearing may lead to surface or sub-surface cracks, as well as cracking in the core of cast products. Hot tearing is also a problem encountered in welding processes, for which it is named "solidification cracking" [2] and should not be confused with "liquation cracking", which is a defect due to the melting of segregated regions with lower melting point around the fusion zone.

Several physical phenomena leading to hot tears (deformation, liquid feeding, wetting, microsegregation) are coupled. This explains that the material susceptibility depends on the alloy composition and microstructure. Numerical simulations have been developed recently, at the scale of a representative elementary volume of the mush $[3,4,5]$. Indeed, in such simulations, the concurrent phenomena have to be modeled at the microscopic scale, but some of them are still insufficiently understood. This is why, for analyzing industrial applications, in which the key problem is the definition of process actuators in order to minimize hot tearing risks, macroscopic criteria are generally considered. Numerous criteria have been proposed in the literature to predict hot tears from the results of a macroscopic thermal-mechanical analysis, as reported in the review of Eskin and Katgerman [6] for instance. Among them, some criteria will be shortly reviewed in the next section. 
Insert Figure 1 (double column format)

The development of a hot tearing criterion in order to predict at least the tendency to hot tearing is currently of great interest for the steel industry, to meet productivity and quality demands, as well as the development of new steel grades. The main objective of this study is to provide a critical assessment of the current possibilities of predicting cracking with such criteria.

The approach consists in selecting two hot tearing tests, and in comparing the experimental results - in terms of occurrence and location of cracks - to the predictions provided by their thermal-mechanical analysis and the use of criteria for crack initiation. Regarding hot tearing tests, a great number of testing devices have been proposed in the past (see for instance the comprehensive review of Eskin et al. [3]). In this study, two hot tearing tests have been selected: a constrained shrinkage test, and an ingot bending test.

The paper is organized as follows. In a first part, a short review of the main hot tearing criteria is presented. Then, the two next sections are dedicated to the presentation and study of the two tests selected. It will be seen that the analysis of the first test (constrained shrinkage) will allow for a first discriminating critical assessment of the different criteria, the best results being obtained with the so-called WYSO criteria proposed by Won et al. [8]. Then, a more quantitative assessment of the predictions of this criterion through the analysis of the ingot bending test will lead the authors to a discussion regarding its merits and limitations. In a final section, an extension of the WYSO criterion is discussed and a new criterion for hot tearing is formulated.

\section{HOT TEARING MACROSCOPIC CRITERIA}

Several criteria aiming at the prediction of crack initiation have been proposed since the early sixties [6]. They all are macroscopic criteria, i.e. they are based on the values of averaged fields at the scale of a representative elementary volume of the mushy zone, such as temperature, plastic strain and strain-rate, stress... The three main classes of criteria are listed hereunder.

\section{Criteria based on thermal considerations only}

The criterion of Clyne and Davies [9], labeled CD in the sequel, mainly takes into account the time spent in a vulnerable state, during which hot tears may develop. The hot cracking sensitivity is defined by:

$$
H C S_{C D}=\frac{t_{V}}{t_{R}}
$$

where $t_{V}$ is the time spent between the critical solid fractions 0.90 and 0.99 , and $t_{R}$ is the time spent between solid fractions 0.40 and 0.90 .

\section{Criteria based on solid mechanics}

Most criteria of this class consider either the strain or the strain rate as damaging variables. It is worth noting that most of the time, the quantities $\varepsilon$ (strain) or $\dot{\varepsilon}$ (strain-rate) used in the expression of the criteria are not precisely defined. A first interpretation could be the norm, in the von Mises sense, of strain $\left(\sqrt{2 / 3} \varepsilon_{i j} \varepsilon_{i j}\right)$, or strain-rate $\left(\sqrt{2 / 3 \dot{\varepsilon}_{i j} \dot{\varepsilon}_{i j}}\right)$. However, authors generally consider that the effective damaging component is the one acting perpendicular to the dendritic columnar growth direction. This is why in this work, the notations $\hat{\varepsilon}$ and $\hat{\dot{\varepsilon}}$ will be used for the damaging strain and the damaging strain-rate, respectively. Those two scalars are defined as a norm of the components of strain (or strain-rate) acting in the plane orthogonal to the dendritic columnar growth direction, as explained in the Appendix.

The criterion of Prokhorov [10], labeled PRO in this paper, can then be expressed as:

$$
\begin{aligned}
& H C C_{P R O}=\operatorname{Max}_{B T R}\left(\hat{\dot{\varepsilon}}-\hat{\dot{\varepsilon}}_{c}\right) \\
& \text { with } \quad \hat{\dot{\varepsilon}}_{c}=D_{\min } \frac{|\dot{T}|}{B T R}
\end{aligned}
$$

This criterion is based on the comparison between the damaging strain-rate $\hat{\dot{\varepsilon}}$ and the admissible strain-rate in the solidifying material $\hat{\dot{\varepsilon}}_{c} \cdot D_{\min }$ is the minimum fracture strain in the BTR, $\dot{T}$ the cooling rate, and BTR the amplitude of the brittleness interval (expressed in ${ }^{\circ} \mathrm{C}$ ).

The criterion of Yamanaka et al. [11], labeled YAM, also relies on a purely mechanical analysis since it compares the accumulated strain undergone by the material over the brittleness temperature range, to a given strain limit. The criterion of Won et al. [8], labeled WYSO, is an extension of the previous one (YAM), including strain-rate influence. It is expressed as follows:

$$
\begin{aligned}
& H C C_{W Y S O}=\operatorname{Max}_{B T R}\left(\int \hat{\dot{\varepsilon}}(t) d t-\hat{\varepsilon}_{c}\right) \\
& \text { with } \hat{\varepsilon}_{c}=\frac{\varphi}{(\hat{\dot{\varepsilon}})^{n^{*}} B T R^{n^{*}}}
\end{aligned}
$$

where the three parameters have been deduced by a non-linear data fitting covering numerous tests performed on different mid-alloyed steel grades: $\varphi=0.02821, m^{*}=0.3131, n^{*}=$ 0.8638 . In the case of the YAM criterion, the strain limit $\hat{\varepsilon}_{c}$ is considered as a material data.

\section{Criteria based on solid and fluid mechanics}


The criterion from Rappaz et al. [12], labeled RDG, assumes that hot tears form when the local interdendritic liquid feeding, through the permeable mushy zone, is not sufficient to compensate the opening of the dendritic network. The combination of mass conservation with Darcy's law and their integration along the length of the mushy zone leads to the expression of a critical damaging strain-rate:

$$
\begin{aligned}
& H C C_{R D G}=\operatorname{Max}_{B T R}\left(\hat{\dot{\varepsilon}}-\hat{\dot{\varepsilon}}_{c}\right) \\
& \text { with } \quad \hat{\dot{\varepsilon}}_{c}=\frac{1}{R}\left[\frac{\lambda_{2}^{2}\|\nabla T\|}{180 \mu_{l}} \frac{\rho_{L}}{\rho_{S}}\left(p_{m}-p_{c}\right)-v_{T} \frac{\rho_{S}-\rho_{L}}{\rho_{S}} H\right]
\end{aligned}
$$

in which $\mu_{l}$ is the dynamic viscosity of the liquid phase, $\lambda_{2}$ is the secondary dendrite arm spacing, $p_{m}$ is the local pressure in the liquid ahead of the mushy zone, $p_{c}$ is the cavitation pressure, $v_{T}$ is the velocity of the solidification front. The quantities $R$ and $H$ depend on the solidification path of the alloy:

$$
\begin{aligned}
& H=\int_{T_{2}}^{T_{1}} \frac{g_{s}{ }^{2}}{g_{l}{ }^{2}} d T \quad R=\int_{T_{2}}^{T_{1}} \frac{g_{s}{ }^{2} F(T)}{g_{l}{ }^{3}} d T \\
& F(T)=\frac{1}{\|\nabla T\|} \int_{T_{2}}^{T} g_{s} d T
\end{aligned}
$$

The integration limits of the integrals are the temperature limiting the BTR.

\section{FIRST HOT TEARING TEST: CONSTRAINED SHRINKAGE}

\section{Experimental: the "Crickacier" hot tearing test}

The "Crickacier" hot tearing test has been developed by the authors and is presented in details in [13,14]. It is basically a constrained shrinkage test (see Figure 2 and Figure 3). Two water-cooled chills are placed at both ends of the cast specimen, initiating first solidification around them. The cast part has the shape of two cone trunks (total length $200 \mathrm{~mm}$ ), such that the section of the specimen is bigger in the mold center in order to generate a central hot spot. During solidification, the fixed solidified regions at the ends generate tensile stresses in the direction perpendicular to the columnar growth direction along the surface of the specimen, in the central hot spot region. It will be seen below that according to the hot spot configuration (that is its shape and the applied mechanical load), cracks may form.

\section{Insert Figure 2}

\section{Insert Figure 3}

The control of the thermal evolution is achieved by three actuators: an insert in the central part of the mold, made of ceramic or steel; pre-heating of the mold; use of ceramic fiber paper along the mold surface.

Two steel grades have been studied: 40CrMnMo8: $0.4 \mathrm{C}$ $2 \mathrm{Cr}-1.5 \mathrm{Mn}-0.3 \mathrm{Ni}-0.2 \mathrm{Mo}$ (wt $\%)$ and $100 \mathrm{Cr} 6: 1.0 \mathrm{C}-1.5 \mathrm{Cr}$. Casting temperature ranged from 1520 to $1620^{\circ} \mathrm{C}$, initial mold temperature from 250 to $425{ }^{\circ} \mathrm{C}$, leading to solidification times between 25 and $150 \mathrm{~s}$.

This test proved to be discriminating as hot tears occurred depending upon the configuration (shape and mechanical load) of the central hot spot. The basic configuration (central insert made of steel, no ceramic paper) left the specimen uncracked. When using a refractory central insert, cracks initiated at the center of the specimen, in the bottom part of the periphery of the transverse medium section. When using in addition a ceramic fiber paper, tears developed in the centre and also near the junction between part and feeder. Those findings are summarized in Figure 4.

\section{Insert Figure 4}

A macrography of one of the central cracks can be observed in Figure 5. Close to the bottom of the part (region I on Figure 5a), the crack is rather rectilinear. A fractography obtained by a scanning electron microscope (Figure 5b) highlights its interdendritic character and its orientation along the direction of growth of the columnar dendrites. This reinforces the hypothesis of crack initiation in region $I$ as a result of the opening of the dendritic network under tensile stress acting perpendicular to the growth direction.

\section{Insert Figure 5}

\section{Numerical simulation: thermal-mechanical finite element modelling}

The finite element modeling of the test has been carried out using the simulation software package THERCAST ${ }^{\circledR}$ [1517]. The numerical simulation consists of a thermal-mechanical stress/strain analysis. At each time increment, the thermal and the mechanical problems are solved sequentially. First, the energy conservation is solved on the whole casting system (the casting and the different mold components illustrated in Figure $2)$. In this resolution, the solidification path $g_{l}(T)$ is defined a priori. The heat exchange coefficient governing the heat transfer between the casting and the mold is defined as a function of the size of the air gap resulting from the mechanical calculation [13]. In a second step, the mechanical problem is solved. The alloy is modeled as follows, using a hybrid constitutive equation [18]. Over the solidus temperature, steel is considered as a non-Newtonian fluid obeying a temperaturedependent viscoplastic Norton-Hoff law:

$$
\bar{\sigma}=K \dot{\bar{\varepsilon}}^{m}
$$

where $\bar{\sigma}$ is the equivalent von Mises stress, $K$ is the viscoplastic consistency, $\dot{\bar{\varepsilon}}$ is the generalized strain rate, and $m$ 
the strain rate sensitivity. The value of $m$ is equal to 1 over the liquidus (Newtonian fluid) and is constantly decreasing with the solid fraction $g_{s}$ in the solidification interval. Below the solidus temperature, in the solid state, the material obeys an elastic-viscoplastic constitutive equation:

$$
\bar{\sigma}=\sigma_{y}+K^{\prime} \bar{\varepsilon}^{n} \dot{\bar{\varepsilon}}^{m}
$$

where $\sigma_{y}$ denotes the plastic yield stress, $\bar{\varepsilon}$ the cumulated plastic strain, and $n$ the strain hardening coefficient.

The thermal-mechanical modeling of the "Crickacier" hot tearing test is illustrated in Figure 6. Accounting for symmetry, one quarter of the set-up is modeled, with a total number of 170000 elements. All rheological parameters appearing in Eqs. (6) and (7), together with elastic coefficients are defined as functions of temperature and can be found in [13] for the two steel grades considered. Also, initial and boundary conditions used for the different casting conditions used for the two steel grades can be found in the same reference.

\section{Insert Figure 6}

Preliminary to this study, the different cracking criteria mentioned above were implemented in the finite element code. The values of the hot cracking criteria are calculated at nodes. The damaging strain-rate is calculated as explained in the Appendix. Regarding the implementation of the RDG criterion, the value of $p_{m}$, the local pressure in the liquid ahead of the mushy zone, is estimated as the nominal initial liquid pressure: $p_{m}=\rho g h$, with $\rho$ the density, $g$ the norm of the gravity vector, and $h$ the vertical distance to the free surface in the riser.

The objective was then to compare the calculated distributions of hot cracking sensitivity produced using those different criteria and the experimental occurrence of cracking in the different cast specimens. As reported in [14], and with full details in [13] the main outputs of the comparison between experimental analyses (crack opening or not, according to the different conditions) and the numerical simulation are the following.

Experimental results clearly show a strong influence of the presence of the ceramic insert on the occurrence of cracking: a steel insert results in no tear, while a refractory insert often leads to cracked parts. Among the different criteria tested, only the $\mathrm{CD}$ criterion does not match with this marked trend.

The analysis of the location of the cracks reveals that the best response is obtained with the criteria based on solid mechanics, namely PRO and WYSO. This is illustrated in Figure 7 and Figure 8 for the two steel grades tested. The predictions of the $\mathrm{CD}$ criterion are wrong, which expresses the strong limitations of this criterion, based on thermal considerations only. The predictions of the RDG criterion are in significant disagreement with experimental findings. It can be noted that according to this criterion, the highest values of hot cracking risk are found at the heart of the central region of the specimen. This reveals the real nature of the RDG criterion, which is basically a porosity criterion: hot tears are supposed to be initiated by liquid film cavitation, resulting from insufficient liquid feeding. Globally, for the two steel grades studied, the best response is obtained with the WYSO criterion, as shown in Figure 9 and Figure 10.

\section{Insert Figure 7}

\section{Insert Figure 8}

\section{Insert Figure 9}

\section{Insert Figure 10}

\section{SECOND HOT TEARING TEST: INGOT BENDING}

A second test has been investigated in this study: an ingot bending test. It consists in bending a $300 \mathrm{~kg}$ steel ingot $(1200 \mathrm{x}$ $140 \times 240 \mathrm{~mm}^{3}$ ) during its solidification (Figure 11a). This test was developed in the 80's at IRSID [18] and was carried out for nine steel grades. The solidified thickness of the ingot was controlled, and a 3-point type flexion test was performed by means of a bending tool. This bending tool consisted of a cylindrical punch, whose displacement and speed (ranging from 0.2 to $5.5 \mathrm{~mm} / \mathrm{s}$ ) were monitored in order to apply different bending strains and strain-rates. Depending on bending conditions, the post-mortem inspections revealed (or not) cracks initiated in the solidification front opposite to the bending tool. The cracks were observed in the region represented in Figure 11b.

\section{Insert Figure 11}

As explained in Figure 12, for each given set of testing conditions (a given steel grade, a given tool speed), repeated tests up to different applied maximum strains revealed that cracks only appeared after a given punch displacement. As explained in [18], the mechanical analysis of numerous tests allowed Bobadilla et al. to derive Bending Limit Curves (BLC), separating in the space $(\hat{\varepsilon}, \hat{\dot{\varepsilon}})$ non-cracked from cracked specimens, for each of the nine steel grades investigated (Figure 13).

\section{Insert Figure 12}

\section{Insert Figure 13}

A first conclusion that can be drawn from those experiments is that there is a strain limit before crack initiation. In other words, below a given strain, there is no crack opening. This important feature allows us to discriminate between the 
different criteria proposed in the literature. Indeed, criteria that are based on a critical strain-rate are certainly not in agreement with the results of Figure 13. This is the case of the PRO and RDG criteria, which do not account for such a strain limit before effective cracking.

A second important remark induced by Figure 13 is that this strain limit depends on strain-rate: the higher the applied strain-rate, the smaller the strain limit. This means that the YAM criterion for instance is not appropriate, because it does not account for such a strain-rate effect.

At this stage, considering the different criteria reviewed above, the only eligible candidate would be the WYSO criterion. The next section is dedicated to a more detailed evaluation of the WYSO criterion through such ingot bending tests.

Assessment of the WYSO criterion through ingot bending

For each bending test, defined by a steel grade and a nominal bending strain-rate, the following two quantities are compared:

○ The strain limit as deduced from the experiment, that is the strain limit separating cracked ingots from noncracked ones. This experimental strain limit is denoted $\hat{\mathcal{\varepsilon}}_{c}^{E X P}$.

- The strain limit corresponding to the WYSO criterion:

$$
\hat{\varepsilon}_{c}^{W Y S O}=\frac{\varphi}{(\hat{\dot{\varepsilon}})^{m^{*}} B T R^{n^{*}}}
$$

where $\hat{\dot{\varepsilon}}$ is the nominal damaging strain-rate of the considered test.

This comparison is given in Figure 14.

\section{Insert Figure 14}

It can be seen that the strain limit provided by the WYSO criterion systematically exceeds the experimental strain limit. After checking the validity of the calculation of the experimental deformations [13], it was suspected that this bias could come from the expression of the BTR, which is the only material-dependent parameter in the expression of the WYSO criterion, since the parameters $\varphi, m^{*}$ and $n^{*}$ are supposed to hold for a wide family of mid-alloyed carbon alloys. Indeed, in this first comparison (Figure 14), thermodynamic equilibrium conditions were assumed to estimate the BTR. In order to check the influence of the BTR, this one has been calculated for five among the nine steel grades considered, using the microsegregation software CEQCSI [19], considering non equilibrium conditions with a prescribed solidification time of $500 \mathrm{~s}$, which is representative of the solidification rate encountered in the ingot tests. New theoretical strain limits could then be calculated and compared to the experimental strain limits. This comparison is given in

Figure 15.
Insert

Figure 15

The confrontation of Figure 14 and

Figure 15 shows a huge influence of the BTR value on the predicted strain limits predicted with the WYSO criterion. This raises two problems:

- First, the calculation of the solidification path $g_{l}(T)$, from which the BTR value is derived, is clearly critical. This is a problem from the user point of view, since the prediction of the WYSO criterion could be significantly different when using different microsegregation models (THERMOCALC, CEQCSI, or any other model).

- Second, and in relation with the first point, the definition itself of the BTR may have a large influence on the value injected in the WYSO criterion, and may then result in very different values of theoretical strain limits.

An example of this second limitation is given in Figure 16. Steel grade 4 is considered. In Figure 16a, the liquid fraction is plotted vs temperature. Three different values of the BTR are considered, corresponding to three couples of critical solid fractions defining this brittleness interval. The results are summarized in Table 1. It can be seen that a small change in the upper bound to define the brittleness interval - expressed in terms of solid fractions - results in a large variation of the BTR value, and consequently in a significant change in the theoretical strain limit.

\section{Insert Figure 16}

\begin{tabular}{|c|c|c|l|}
\hline$g_{s} \min$ & $g_{s} \max$ & $B T R\left[{ }^{\circ} \mathrm{C}\right]$ & \multicolumn{1}{|c|}{$\hat{\varepsilon}_{c}^{\text {WYSO }}$} \\
\hline 0.9 & 0.97 & 41 & $\operatorname{ref} \times 1.41$ \\
\hline 0.9 & 0.99 & 57 & ref \\
\hline 0.9 & 0.999 & 97 & $\operatorname{ref} \times 0.64$ \\
\hline
\end{tabular}

Table 1. BTR values corresponding to different characteristic solid fractions used to estimate the brittleness domain.

At this stage, the main conclusions that can be drawn from the assessment of the WYSO criterion through the analysis of "Crickacier" and ingot bending experimental tests are as follows:

- The WYSO criterion offers a very good qualitative response in terms of sensitivity to solidification conditions and prediction of possible crack initiation. This is clearly illustrated by the "Crickacier" and ingot bending tests.

- However, the application to ingot bending, for which a more quantitative analysis is possible, reveals that a direct 
application of the WYSO criterion is not in quantitative agreement with experimental measurements.

- This is due to the fact that the BTR value is actually the only variable in the expression of the WYSO criterion that is related to steel composition.

- The BTR itself cannot account for the observed influence of chemical elements such as carbon, sulfur and phosphorus on hot cracking sensitivity [20]. These last two elements diffuse slowly in ferrite and austenite and have low partition coefficients, which favors interdendritic segregation [21].

- The calculation of the BTR itself is a real issue. Given the important role of the above mentioned elements, the solidification path must be calculated in non-equilibrium conditions, but such estimates significantly depend on the microsegregation model used.

- Furthermore, in the case of a solidification path calculated in non-equilibrated conditions, the BTR range is extremely sensitive to the choice of the two solid fraction bounds.

\section{A NEW CRITERION}

In this section, a modification of the WYSO criterion is proposed and tested. Actually, despite its limitations, the WYSO criterion presents significant advantages. First, it offers a good response, which has been confirmed by the present study in different solidification conditions and for different steel grades. Second, it is quite suited for implementation in thermal-mechanical finite element codes which are routinely used for the analysis of industrial solidification processes. The main advantage of this criterion is that it is based on a single material-dependant parameter, the BTR. But this simplicity is also the source of the limitations. Therefore, there is a strong motivation to propose an extension of this criterion, keeping its simplicity and overcoming its limitations. The subsequent modifications of the WYSO criterion are suggested, to construct a new criterion, which will be named CBC criterion.

The driving idea consists in expressing the strain limit not only with the sole BTR as material dependent parameter, like in the original WYSO criterion, but with an additional parameter depending on the steel grade. In order to proceed to this modification, the hot tearing index (WF) of Wünnenberg and Flender [22] has been considered. This index was initially proposed to rank steel grades with respect to their susceptibility to hot tearing, and has the following expression:

$$
I_{W F}=a_{0} \exp \left(b_{0} \mathrm{wt} \% \mathrm{C}\right)\left(\frac{\mathrm{wt} \% \mathrm{Mn}}{\mathrm{wt}^{2} \% \mathrm{~S}}\right)^{c_{0}}
$$

with $a_{0}=30.848 ; b_{0}=2.195 ; c_{0}=-0.857$.

It is interesting to note the values of this index for the nine steel grades considered in the present study (Table 2).

\begin{tabular}{|c|c|c|c|}
\hline $\begin{array}{c}\text { Steel } \\
\text { grade }\end{array}$ & $\mathrm{wt} \% \mathrm{C}$ & $\begin{array}{c}\mathrm{wt} \% \mathrm{Mn} / \mathrm{wt} \% \\
\mathrm{~S}\end{array}$ & $I_{W F}$ \\
\hline 1 & 0.11 & 82 & 0.9 \\
\hline 2 & 0.11 & 36 & 1.9 \\
\hline 3 & 0.18 & 65 & 1.3 \\
\hline 4 & 0.20 & 27 & 2.8 \\
\hline 5 & 0.17 & 5 & 11.3 \\
\hline 6 & 0.41 & 92 & 1.6 \\
\hline 7 & 0.36 & 27 & 4.0 \\
\hline 8 & 0.15 & 79 & 1.0 \\
\hline 9 & 0.40 & 92 & 1.5 \\
\hline
\end{tabular}

Table 2. Hot tearing index of Wünnenberg and Flender for the nine steel grades studied.

In order to establish a correlation between this hot tearing index $\left(I_{W F}\right)$ and the hot tearing criterion $\left(H C C_{W Y S O}\right)$, we have proceeded as follows. For any combination of two steel grades (let us denote them G1 and G2) taken from the nine steel grades studied (there are 36 possible combinations), and given a selected nominal strain-rate of $1 \times 10^{-3} \mathrm{~s}^{-1}$, the following two ratios have been calculated:

- $\hat{\varepsilon}_{c G 1} / \hat{\varepsilon}_{c G 2}$, in which the two quantities are calculated using Eq. (8), with $\hat{\dot{\varepsilon}}=1 \times 10^{-3} \mathrm{~s}^{-1}$.

○ $I_{W F_{G} G 2} / I_{W F_{-} G 1}$, in which the two quantities are calculated using Eq. (9).

Figure 17 shows the first ratio vs the second one, for each of the 36 possible combinations.

\section{Insert Figure 17}

In this figure, the correlation between the considered hot tearing criterion (WYSO) and the hot tearing index of Wünnenberg and Flender is shown. However, it is worth noting that this correlation is not so good for the high-P grades (grades 8 and 9 , indicated by a ' $X$ ' mark).

The physical analysis of this correlation is the following. Expression (9) shows that carbon and sulfur (when not neutralized by manganese by formation of $\mathrm{MnS}$ ) increase the hot tearing susceptibility. Regarding sulfur, the partition coefficient between solid and liquid phases is small, which favors microsegregation and tends to decrease the complete solidification temperature, that is to increase the BTR $[8,20$, 21]. Note also that the surface tension of the liquid phase decreases with the sulfur content [23], which favors the wetting of the solid phase by the liquid phase, and then increases the hot tearing susceptibility [24]. The negative influence of carbon, can be associated with microsegregation, leading to increased BTR with higher carbon content, even if this effect is tempered by the occurrence of the peritectic reaction in the range 0.08 to $0.16 \mathrm{wt} \%$ [8]. It may also come from the decrease of the surface tension of the liquid phase with the carbon content [23]. Finally, the role of phosphorus is ignored by 
expression (9), which is probably the cause of the deviation noted in Figure 17. Indeed, as reported by [8, 20], phosphorus has a significant effect on the BTR by microsegregation effect (low partition coefficient), and then a detrimental effect on hot cracking resistance.

At this stage, it is noted that microsegregation explains - at least partially - the fact that hot tearing susceptibility increases with carbon, sulfur and phosphorus contents. Compared to the BTR value that would result from equilibrium solidification conditions, that is without segregation effect, the BTR value calculated in non-equilibrium conditions may be significantly larger, thus yielding smaller values of the strain limit, according to the WYSO expression (8). As a consequence, it is proposed here to proceed to the following decoupling. On one hand, the original expression of the criterion (8) is conserved, but including a BTR value that should be calculated in equilibrium conditions. On the other hand, the influence of the chemical species $\mathrm{C}, \mathrm{S}$ and $\mathrm{P}$ is taken into account by a multiplier which acts as a penalty term. This term can be derived from the WF index, by taking its inverse and including the influence of the phosphorus content. Finally the new proposed expression for the $\mathrm{CBC}$ criterion is defined as follows:

$$
\begin{aligned}
& H C C_{C B C}=\operatorname{Max}_{B T R_{e q}}\left(\int \hat{\dot{\varepsilon}}(t) d t-\hat{\varepsilon}_{c}\right) \\
& \hat{\varepsilon}_{c}=\frac{\varphi}{(\hat{\dot{\varepsilon}})^{m^{*}} B T R_{e q}^{n^{*}}} \frac{1}{a \exp \left(b_{0} \mathrm{wt} \% \mathrm{C}\right)\left(\frac{\mathrm{wt} \% \mathrm{Mn}}{\mathrm{wt} \% \mathrm{~S}}\right)^{c_{0}}(\mathrm{wt} \% \mathrm{P})^{d}}
\end{aligned}
$$

The parameters of the initial criterion are kept unchanged: $\varphi=0.02821, m^{*}=0.3131, n^{*}=0.8638$, and $B T R_{e q}$ denotes the value of the BTR calculated in equilibrium condition. The parameters $b_{0}$ and $c_{0}$ are kept unchanged, while the parameter $a_{0}$ needs to be calibrated, and a complementary parameter $d$ is introduced. Note that expression (10b) can be seen as an extension of the WYSO expression, by introducing the new function $\varphi^{\prime}$ in place of the original parameter $\varphi$, expressing its dependence with respect to chemical composition:

$$
\varphi^{\prime}=\frac{\varphi}{a \exp \left(b_{0} \mathrm{wt} \% \mathrm{C}\right)\left(\frac{\mathrm{wt} \% \mathrm{Mn}}{\mathrm{wt} \% \mathrm{~S}}\right)^{c_{0}}(\mathrm{wt} \% \mathrm{P})^{d}}
$$

The application of this new CBC criterion to the ingot bending test has provided the best comparison with the experimental strain limit for:

- BTR value calculated in equilibrium conditions for solid fraction bounds of 0.85 and 0.97 ;

○ $a=736.5, d=0.851$.

This comparison is shown in Figure 18. There is a good overall agreement between the estimated strain values, corresponding to the experimental BLCs, and the new proposed expression of the critical strain (Eq. (10)), thus validating this new approach. However, it can be seen that the accuracy of the quantitative prediction of the new criterion varies with the different steel grades tested. This probably reflects the fact that despite its merits, the proposed criterion does not encompass all the complexity of the concurrent physics resulting in hot tearing phenomena.

\section{Insert Figure 18}

\section{CONCLUSION}

A critical assessment of several hot tearing criteria has been carried out using two hot tearing experimental tests: the "Crickacier" constrained solidification test developed at Cemef [14], and the ingot bending test formerly developed and applied at IRSID [18]. Comparisons between thermal-mechanical analysis and experimental findings have resulted in a discriminating screening of different criteria proposed in the literature. The conclusion drawn is that the more reliable criterion regarding the sensitivity to solidification conditions and the prediction of cracking location is the so-called WYSO criterion initially proposed by Won et al. [8]. A detailed analysis of the ingot bending test, which allows for an effective quantitative evaluation of the damaging strains and strain-rates, shows that the strain limit as predicted by the WYSO criterion systematically exceeds experimental estimates. Besides, some severe limitations of the WYSO criterion arise from the determination of the brittleness temperature range in non equilibrium conditions. To overcome those difficulties, the original criterion has been reformulated, expressing the BTR in equilibrium conditions and adding some new terms associated with some critical chemical elements such as S, Mn, C and P. The application of this new $\mathrm{CBC}$ criterion to ingot bending tests performed on nine steel grades shows a better quantitative prediction. Because of this decoupling between BTR and critical chemical elements, the predictions of the $\mathrm{CBC}$ criterion are less dependent of the choice and mode of use of a microsegregation model. Combined with macroscopic thermalmechanical simulations of casting processes, this new criterion should offer extended hot tearing prediction capacities. However, as shown through this comparison exercise, more fundamental work is needed to develop prediction tools that would be based upon a complete integration of the complex physics of hot tearing.

\section{ACKNOWLEDGMENTS}

This study has been supported by the different partners of the Crickacier project: Arcelor Research (ArcelorMittal group), Ascometal (Lucchini group), Aubert et Duval, and Industeel (ArcelorMittal group). 


\section{REFERENCES}

1. J. Campbell: Castings, Butterworth-Heinemann, Oxford, 1991.

2. T. Böllinghaus and H. Herold (eds.): Hot Cracking Phenomena in welds, Springer, Berlin, 2005.

3. S. Vernède, Ph. Jarry and M. Rappaz: Proc. $11^{\text {th }}$ Int. Conf. on Modeling of Casting, Welding and Advanced Solidification Processes, Ch.-A. Gandin and M. Bellet (eds.), TMS, 2006, pp. 635-42.

4. D. Fuloria, P.D. Lee and D. Bernard: Proc. SP07, $5^{\text {th }}$ Decennial Int. Conf. on Solidification Processing, Sheffield, H. Jones (ed.), The University of Sheffield, 2007, pp. 685-89.

5. A.B. Phillion, S.L. Cockroft and P.D. Lee: Acta Materialia, 2008, vol. 56, pp. 4328-38.

6. D.G. Eskin and L. Katgerman: Met. Mater. Trans. A, 2007, vol. 38A, pp. 1511-19.

7. D.G. Eskin, Suyitno and L. Katgerman: Progress in Materials Science, 2004, vol. 49, pp. 629-711.

8. Y.M. Won, T.J. Yeo, D.J. Seol and K.H. Oh: Metall. Mater. Trans. B, 2000, vol. 31B, pp. 779-94.

9. T.W. Clyne and G.J. Davies: Solidification and casting of metals, The Metals Society, 1977, pp. 275-78.

10. N.N. Prokhorov: Russian Castings Production, 1962, vol. 2, pp. 172-75.

11. A. Yamanaka, K. Nakajima, K. Yasumoto, H. Kawashima and K. Nakai: Proc. $5^{\text {th }}$ Int. Conf. on Modeling of Casting, Welding and Advanced Solidification Processes, M. Rappaz, M.R. Ozgu and K.W. Mahin (eds.), TMS, 1991, pp. 279-84.

12. M. Rappaz, J.M. Drezet and M. Gremaud: Metall. Mater. Trans. A, 1999, vol. 30A, pp. 449-56.

13. O. Cerri: Rupture à chaud dans les aciers au cours de leur solidification, caractérisation expérimentale et modélisation thermomécanique (Hot tearing in steels during solidification, experimental characterization and thermal-mechanical modelling), Ph.D. Thesis, MinesParisTech, 2007, http://pastel.paristech.org/.

14. O. Cerri, Y. Chastel and M. Bellet: ASME J. Eng. Mat Tech., 2008, vol. 130, pp. 1-7.
15. http://www.transvalor.com/thercast_gb.php, 2009.

16. M. Bellet, O. Jaouen and I. Poitrault: Int. J. Num. Meth. Heat Fluid Flow, 2005, vol. 15, pp. 120-42.

17. M. Bellet and V.D. Fachinotti: Comput. Methods Appl. Mech. and Engrg., 2004, vol. 193, pp. 4355-81.

18. M. Bobadilla, B. Chamont, C. Gatellier and J.M. Jolivet: European Community Assembly, Convention 7210CA/316, RE 88/023, 1988.

19. M. Wintz, M. Bobadilla and J. Lehmann: Proc. $4^{\text {th }}$ Decennial Int. Conf. on Solidification Processing, J. Beech and H. Jones (eds.), The University of Sheffield, 1997, pp. 226-29.

20. M. Wintz, M. Bobadilla and J.M. Jolivet: La revue de Métallurgie, 1994, vol. 4, pp. 105-14.

21. Y.M. Won, H.N. Han, T.J. Yeo and K.H. Oh: ISIJ Int., 2000, vol. 40, pp. 129-36.

22. K. Wünnenberg and R. Flender: Ironmaking and Steelmaking, 1985, vol. 12, pp. 22-29.

23. V.N. Saveiko: Russian Castings Production, 1961, vol. 11, pp. 453-56.

24. J.C. Borland: Br. Welding J., 1960, vol. 7, pp. 508-12. 


\section{APPENDIX: Definition of damaging strain and strain- rate}

In this study, it is suggested to take into account the orientation of the mechanical loading mode with respect to the microstructure topology in the expression of the different hot tearing criteria. For that purpose, the components of the strainrate tensor should be expressed in a reference frame associated with the columnar dendritic growth direction (Figure 19).

\section{Insert Figure 19}

Such a reference frame $(\mathbf{u}, \mathbf{v}, \mathbf{w})$ can be defined as follows:

$$
\left\{\begin{array}{l}
\mathbf{u}=\frac{\nabla T}{\|\nabla T\|} \\
\mathbf{v} / \mathbf{u} \cdot \mathbf{v}=0 \text { and }\|\mathbf{v}\|=1 \\
\mathbf{w}=\mathbf{u} \times \mathbf{v}
\end{array}\right.
$$

Note that there is no uniqueness in this definition, as the orientation of vectors $\mathbf{v}$ and $\mathbf{w}$ is arbitrary. But we will show further that this is not a critical issue. It is now possible to express the strain-rate tensor in this new reference frame:

$$
\dot{\boldsymbol{\varepsilon}}^{(\mathbf{u}, \mathbf{v}, \mathbf{w})}=\mathbf{P}^{\mathrm{T}} \dot{\boldsymbol{\varepsilon}}^{(\mathbf{i}, \mathbf{j}, \mathbf{k})} \mathbf{P}
$$

where $\mathbf{i}, \mathbf{j}$ and $\mathbf{k}$ are the base vectors of the initial reference frame, and $\mathbf{P}$ the rotation matrix between the two reference frames. Consider now the most general expression of the strainrate tensor in this new reference frame:

$$
\dot{\boldsymbol{\varepsilon}}^{(\mathbf{u}, \mathbf{v}, \mathbf{w})}=\left[\begin{array}{lll}
\dot{\varepsilon}_{11} & \dot{\varepsilon}_{12} & \dot{\varepsilon}_{13} \\
\dot{\varepsilon}_{21} & \dot{\varepsilon}_{22} & \dot{\varepsilon}_{23} \\
\dot{\varepsilon}_{31} & \dot{\varepsilon}_{32} & \dot{\varepsilon}_{33}
\end{array}\right]_{(\mathbf{u}, \mathbf{v}, \mathbf{w})}
$$

The tensile (or compression) and shear strain-rate components applied to the material along a plane perpendicular to the growth direction are now contained in the sub-matrix $\mathbf{e}$ :

$$
\mathbf{e}=\left[\begin{array}{ll}
\dot{\varepsilon}_{22} & \dot{\varepsilon}_{23} \\
\dot{\varepsilon}_{32} & \dot{\varepsilon}_{33}
\end{array}\right]_{(\mathbf{v}, \mathbf{w})}
$$

A first proposal for the definition of a scalar quantity representing the damaging strain-rate consists in extracting the two eigen values of this matrix, $\lambda_{1}$ and $\lambda_{2}$. Then, assuming that the damaging components are those of tensile modes, the scalar $\hat{\dot{\varepsilon}}$ can be defined as the larger positive eigen value:

$$
\hat{\dot{\varepsilon}}=\max \left(\lambda_{1}, \lambda_{2}, 0\right)
$$

An alternative definition could consist in taking the von Mises equivalent scalar:

$$
\hat{\dot{\varepsilon}}=\sqrt{\frac{2}{3} \mathbf{e}: \mathbf{e}}=\sqrt{\frac{2}{3} e_{i j} e_{i j}}
$$

Note that expressions (16) and (17) are indifferent to the choice of the two base vectors $\mathbf{v}$ and $\mathbf{w}$ perpendicular to the growth direction. In this work, expression (16) has been preferred. Indeed, it is consistent with the assumption that hot tearing is initiated by fracture of interdendritic liquid films under tension.

Finally, the damaging strain is defined as the timeintegration of $\hat{\dot{\varepsilon}}$.

$$
\hat{\varepsilon}=\int \hat{\dot{\varepsilon}} \mathrm{d} t
$$




\section{FIGURE CAPTIONS}

Figure 1. Schematics of the main phenomena occurring in the mushy zone at characteristic solid fractions, explaining the loss of ductility in a given brittle temperature range.

Figure 2. CAD description of the "Crickacier" hot tearing test developed at Cemef. A quarter of the device is shown. The water-cooled end and the central insert can be distinguished, as well as the location of heating cartridges. Locations labeled $\mathrm{K}$ and $\mathrm{S}$ are those of thermocouples in the mold and in the part, respectively.

Figure 3. The "Crickacier" testing device.

Figure 4. Different schematic solidification configurations and corresponding observed crack locations. Liquid regions are represented in light grey and crack locations are indicated with white lines. a) steel central insert, b) refractory central insert, generating a marked central hot spot, c) intermediate configuration, using refractory central insert and ceramic fiber paper.

Figure 5. a) Macrography of a tear in the transverse symmetry plane of the specimen. The lower surface of the specimen is on the left part of the picture. b) SEM fractography of the tear in the subsurface region of the specimen (zone I of the picture above).

Figure 6. Thermal-mechanical finite element modeling: a) finite element meshes of the different components of the Crickacier set-up; b) longitudinal stress distribution at a given time during solidification, exhibiting tensile stresses perpendicular to the growth direction. The white contours display surfaces of same solid fraction, indicating the location of the mushy zone.

Figure 7. Simulation for the steel grade 40CrMnMo8 (cast with steel insert and ceramic fiber paper). Hot cracking sensitivity (HCS) as predicted using different criteria (CD, WYSO, PRO and RDG).

Figure 8. Simulation for the grade 100Cr6 (cast with steel insert and ceramic fiber paper). Hot cracking sensitivity as predicted using different criteria (CD, WYSO, PRO and RDG).

Figure 9. Comparison between experimental crack location (in the ellipse) and calculated WYSO hot tearing sensitivity for the steel grade $40 \mathrm{CrMnMo} 8$ (cast with steel insert and ceramic fiber paper).

Figure 10. Comparison between experimental crack location (revealed by microscopic observation and chemical penetrant inspection) and calculated WYSO hot tearing sensitivity for the steel grade 100Cr6 (cast with steel insert and ceramic fiber paper).

Figure 11. On the left: the ingot bending test initially developed by Bobadilla et al. [18]. On the right: its thermal-mechanical finite element modeling with THERCAST ${ }^{\mathbb{R}}$. In red, the "zone of interest" is indicated. The distribution of the values of the WYSO criterion is plotted in the mushy zone.

Figure 12. On the left: the procedure of determination of BLCs developed by Bobadilla et al. [18] consisted of repeated tests up to several applied maximum strains, followed up by post-mortem analysis revealing or not crack initiation in the zone of interest. On the right: the bending limit curve (BLC) as determined from several tests at different nominal damaging strain-rates. Superimposed is a 
curve reported from finite element simulation showing that, according to the analysis in [18], the damaging strain-rate remains approximately constant in the zone of interest during a bending test.

Figure 13. Bending Limit Curves as deduced in [18] from ingot bending tests on 9 steel grades.

Figure 14. Comparison between the experimental strain limit (separating cracked to non-cracked ingots) and the theoretical strain limit provided by the WYSO criterion, in which the BTR is deduced from a calculation of $g_{l}(T)$ in equilibrium condition. Comparison on nine steel grades and three nominal strain rates $\left(0.0005,0.001\right.$ and $\left.0.002 \mathrm{~s}^{-1}\right)$.

Figure 15. Comparison between experimental and theoretical strain limits provided by the WYSO criterion, in which the BTR is deduced from a calculation of $g_{l}(T)$ in non-equilibrium condition (CEQCSI microsegregation software, with a prescribed solidification time of $500 \mathrm{~s}$ ).

Figure 16. Influence of BTR definition. a) liquid volume fraction vs temperature, as calculated using CEQCSI microsegregation software for the steel grade 4. b) Zoom on the lower liquid fraction domain, showing three BTR values, for three different definitions of critical liquid fractions (as defined in Table 1).

Figure 17. Correlation between the theoretical strain limit and the hot tearing index of Wünnenberg and Flender. Comparison carried out on all combinations of two steel grades taken from the nine studied steel grades. ' $X$ ' marks correspond to comparisons involving grades with high $\mathrm{P}$ content.

Figure 18. Comparison between the experimental strain limit (separating cracked to non-cracked ingots) and the theoretical strain limit provided by the newly proposed CBC criterion. Comparison on nine steel grades and three nominal strain rates $(0.0005,0.001$ and $\left.0.002 \mathrm{~s}^{-1}\right)$.

Figure 19. Definition of a reference frame $(\mathbf{u}, \mathbf{v}, \mathbf{w})$ associated with the columnar dendritic growth direction. 